\title{
The Time Course Changes in Bone Metabolic Markers after Administering the Anti-Receptor Activator of Nuclear Factor-Kappa B Ligand Antibody and Drug Compliance among Patients with Osteoporosis
}

\author{
Kazuhide Inage ${ }^{1}$, Sumihisa Orita ${ }^{1}$, Kazuyo Yamauchi ${ }^{1}$, Yoshihiro Sakuma ${ }^{2}$, \\ Go Kubota ${ }^{1}$, Yasuhiro Oikawa ${ }^{3}$, Takeshi Sainoh ${ }^{1}$, Jun Sato ${ }^{1}$, Kazuki Fujimoto ${ }^{1}$, \\ Yasuhiro Shiga ${ }^{1}$, Kazuhisa Takahashi ${ }^{1}$, Seiji Ohtori ${ }^{1}$ \\ ${ }^{1}$ Department of Orthopedic Surgery, Graduate School of Medicine, Chiba University, Chiba, Japan \\ ${ }^{2}$ Department of Orthopedic Surgery, National Hospital Organization Chiba Medical Center, Chiba, Japan \\ ${ }^{3}$ Department of Orthopedic Surgery, Teikyo University Chiba Medical Center, Chiba, Japan
}

Study Design: Retrospective study.

Purpose: We conducted a study to investigate the time course changes in bone metabolic markers after the administration of the anti-receptor activator of nuclear factor-kappa B ligand (RANKL) antibody and to assess drug compliance among osteoporotic patients. Overview of Literature: The anti-RANKL antibody is expected to provide an improvement in those with a bone metabolism disorder. However there are only a few clinical reports available on the effect of treatment.

Methods: We included 40 post-menopausal osteoporotic patients who received the anti-RANKL antibody. To determine the time course changes in the bone metabolic markers, we measured the serum tartrate-resistant acid phosphatase $5 b$ (TRACP $5 b$; a bone resorption marker) and the serum N-terminal propeptide of type 1 collagen (P1NP; a bone formation marker) levels prior to and 1 month after administrating the anti-RANKL antibody. To evaluable drug compliance, we assessed the dropout rate during treatment and at 6 months after treatment.

Results: The average TRACP $5 \mathrm{~b}$ level significantly decreased from $574.8 \mathrm{mU} / \mathrm{dL}$ before treatment to $153.2 \mathrm{mU} / \mathrm{dL} 1 \mathrm{month}$ after treatment $(p<0.05)$. There was no significant difference in the average P1NP level, which was $56.9 \mu \mathrm{G} / \mathrm{L}$ and $35.1 \mu \mathrm{G} / \mathrm{L}$ before and 1 month after treatment, respectively $(p>0.05)$. As for drug compliance, we did not have any dropouts during the treatment or after 6 months (dropout rate: $0 \%$ ).

Conclusions: Our study suggests that anti-RANKL antibody treatment suppresses bone resorption and maintains bone formation.

Keywords: Tartrate-resistant acid phosphatase; Receptor activator of nuclear factor-kappa B ligand

\section{Introduction}

Osteoporosis is a primary factor of locomotive syndrome.
The number of osteoporosis patients in Japan has been increasing, mainly in elderly women, and it is estimated that the number may reach 13 million and include non-med-

Received Oct 30, 2014; Revised Nov 25, 2014; Accepted Nov 25, 2014

Corresponding author: Kazuhide Inage

Department of Orthopedic Surgery, Graduate School of Medicine, Chiba University,

1-8-1 Inohana Chuo-ku, Chiba 260-8670, Japan

Tel: +81-43-226-2117, Fax: +81-43-226-2116, E-mail: kazuhideinage@yahoo.co.jp 
ical patients without any subjective symptoms. Among patients aged $\geq 50$ years, it is reported that $14.5 \%$ of males and $51.3 \%$ of females will develop osteoporosis [1]. Various drugs have been used for osteoporosis in recent years and have demonstrated a certain level of effectiveness in improving bone density. However, since these drugs take time to exert its effect or only improves bone density and not bone strength, there are still many uncertainties, such as the influence of the temporal effect on the bone metabolism disorder in osteoporotic patients according to the individual osteoporosis drug [2].

Furthermore, osteoporosis drugs have various disadvantages and adverse effects [3-11]. For instance, heartburn caused by bisphosphonates may cause patients to discontinue oral use. According to a study on the oral use of an osteoporosis drug, $45.2 \%$ of patients were unable to use the drug within 1 year after treatment initiation, and $52.1 \%$ eventually withdrew from treatment within 5 years [12].

However, the antibody against the receptor activator of nuclear factor-kappa B ligand (RANKL), which was recently introduced in Japan, shows a strong inhibitory effect on bone resorption, and improvement is expected in those with a bone metabolism disorder. Drug compliance is expected to be high because it is administered as a hypodermic injection biannually. Since the postintroduction period is still relatively short, there are only a few clinical reports available on the treatment's effect. Therefore, we conducted a study to investigate the time course changes in bone metabolic markers after the administration of the anti-RANKL antibody and to assess drug compliance among osteoporotic patients.

\section{Materials and Methods}

We included 40 post-menopausal osteoporotic patients (mean age \pm standard deviation [SD], $74.7 \pm 7.6$ years) who received anti-RANKL antibody at our medical facility. The inclusion criterion for osteoporosis was a young adult mean (YAM) level $\leq 70 \%$ in accordance with the Japan Osteoporosis Society's guidelines (mean YAM level \pm SD, $64.0 \% \pm 4.4 \%$ ). Patients with a prior history of taking drugs prescribed for osteoporosis were excluded from this study. In addition, MRIs were conducted for all anamnestic cases of external injury. Fresh fracture cases were excluded, as well as anamnestic cases of fragility fracture. We also administered calcium and vitamin $\mathrm{D}$ to all patients.

\section{Primary endpoint}

To determine the time course changes in bone metabolic markers, we measured the serum tartrate-resistant acid phosphatase 5b (TRACP 5b; a bone resorption marker) and N-terminal propeptide of type 1 collagen (P1NP; a bone formation marker) levels before and 1 month after administration of the anti-RANKL antibody. To evaluable drug compliance, we assessed the dropout rate during treatment and 6 months after treatment.

\section{Secondary endpoint}

To evaluate bone density, we measured the lumbar spine YAM level from dual radiography absorptiometry scans before and 6 months after administering anti-RANKL antibody. Additionally, we assessed the time course changes by using the back pain visual analogue scale (VAS) before treatment and at 1,2,3, and 6 months after treatment. Lastly, we measured the serum calcium level before and at 1 week and 1 month after treatment to assess for adverse effects, such as hypocalcemia.

\section{Results}

\section{Primary endpoint}

As shown in Fig. 1, the average TRACP 5b level was significantly decreased from $574.8 \mathrm{mU} / \mathrm{dL}$ before treatment to $153.2 \mathrm{mU} / \mathrm{dL} 1$ month after treatment $(p<0.05)$. The average improvement rate 1 month after treatment was

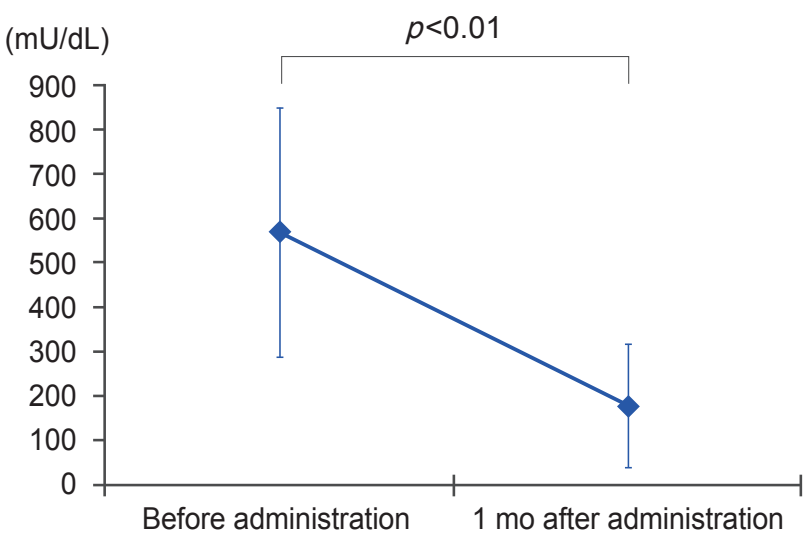

Fig. 1. TRACP $5 b$ levels before and 1 month after administrating the anti-RANKL antibody. TRACP 5b, tartrate-resistant acid phosphatase $5 b$; RANKL, receptor activator of nuclear factor-kappa B ligand. 
$68.2 \%$. There was no significant difference in the average P1NP; before treatment it was $56.9 \mu \mathrm{G} / \mathrm{L}$ and $35.1 \mu \mathrm{G} /$ L 1 month after treatment compared to before treatment as shown in Fig. 2 ( $p>0.05)$. The average lowering rate 1 month after treatment was $22.1 \%$. As for drug compliance, we did not have any dropouts during the treatment or after 6 months (dropout rate: 0\%).

\section{Secondary endpoint}

The average YAM level for the anti-RANKL antibody before and 6 months after treatment was significantly increased from $64.0 \%$ to $69.5 \%$, respectively, with an average increase rate of $8.9 \%$ as shown in Fig. $3(p<0.05)$. The average VAS level for back pain was temporally decreased $(6.2,4.7,3.9,2.9$, and 1.9 before treatment and at 1, 2, 3 , and 6 months after treatment, respectively). In particular,

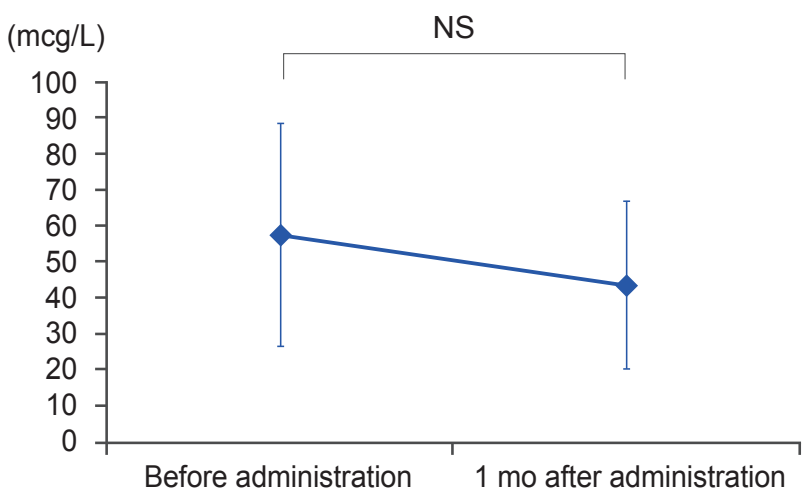

Fig. 2. P1NP levels before and 1 month after administrating the antiRANKL antibody. P1NP, N-terminal propeptide of type 1 collagen; RANKL, receptor activator of nuclear factor-kappa B ligand; NS, not significant.

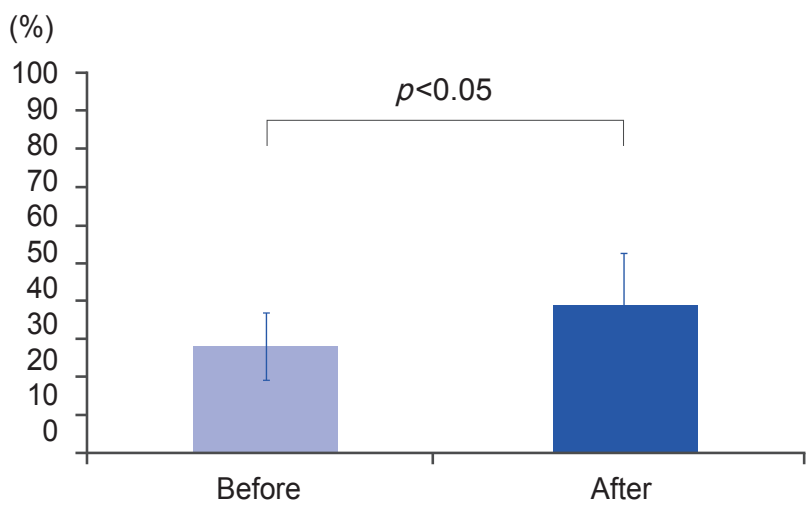

Fig. 3. The averages YAM level for the anti-RANKL antibody before and 6 months after treatment. YAM, young adult mean; RANKL, receptor activator of nuclear factor-kappa B ligand. there was no significant difference between VAS levels before treatment and 1 and 2 months after treatment, but the level was significantly lower 3 and 6 months after treatment as seen in Fig. $4(p<0.05)$. The average calcium levels were 9.4, 9.3, and $9.3(\mathrm{mg} / \mathrm{dL})$ before, 1 week, and 1 month after treatment, respectively, all of which were within the normal range. In addition, these levels were maintained without any significant differences during all the time periods (Fig. 5).

\section{Discussion}

In this study, the average TRACP $5 \mathrm{~b}$ level was significantly lowered by the administration of the anti-RANKL antibody, and the average improvement rate was $68.2 \%$. The improvement rates of bone metabolic markers from commonly used osteoporosis drugs are shown in Table

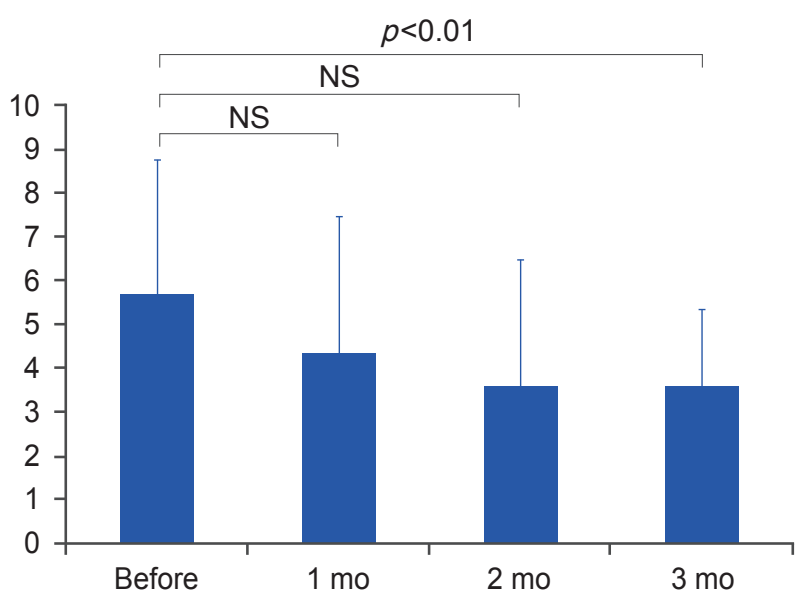

Fig. 4. The time course changes by using the back pain VAS before treatment and 1,2,3, and 6 months after treatment. VAS, visual analogue scale; NS, not significant.

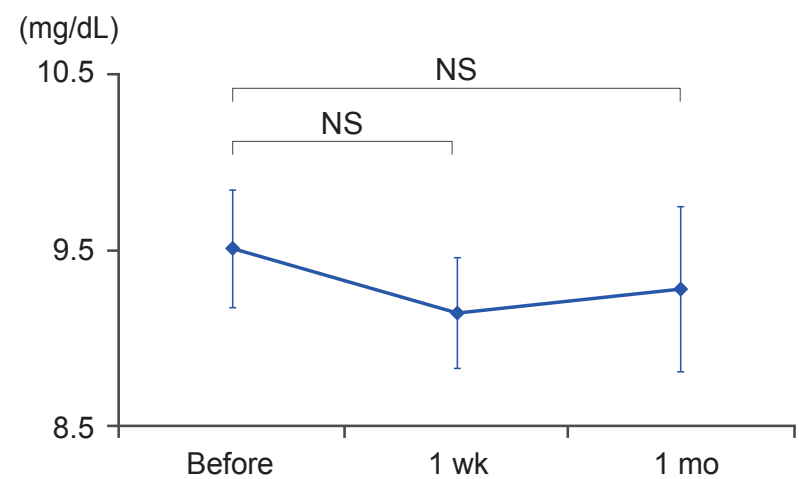

Fig. 5. The serum calcium levels before and 1 week and 1 month after treatment. NS, not significant. 
Table 1. The improvement rates of bone metabolic markers from commonly used osteoporosis drugs

\begin{tabular}{lccc} 
Characteristic & NTX $(\%)$ & BAP (\%) & Periods (mo) \\
Vitamin D & $3-28$ & $15-30$ & 6 \\
SERM & $24-38$ & $24-38$ & 13 \\
Bisphosphonate & $36-52$ & $33-52$ & 13 \\
\hline
\end{tabular}

NTX, N-terminal crosslinking telopeptide of type I collagen; BAP, bone specific alkaline phosphatase; SERM, selective estrogen receptor modulator.

1 [13-17]. We cannot draw a comparison, because different types of bone metabolic markers and followup periods were used, however, the improvement rate of the bone resorption marker was approximately $30 \%$ with vitamin $\mathrm{D}, 40 \%$ with the selective estrogen receptor modulator (SERM), and 50\% with bisphosphonates. The anti-RANKL antibody is a human immunoglobulin G2 monoclonal antibody that targets RANKL and binds to it with a high specificity and affinity. The anti-RANKL antibody binds to RANKL, preventing it from binding to RANK, and also simultaneously suppresses the formation, function, and viability of osteoclasts. Therefore, bone resorption is suppressed and the bond strength is enhanced while the bone mass for cortical and cancellous bones are increased [18]. However, bisphosphonates, which are widely used for osteoporosis treatment, only affect matured osteoclasts, not RANKL. Furthermore, vitamin D and SERM are only involved in the pathological condition of abnormal bone metabolism to a certain extent. The difference in the site of action in this study led to a strong inhibition of bone resorption.

We recognized that the bone formation marker was relatively unchanged despite the administration of the antiRANKL antibody. Generally, a bone formation marker indicates the same rate of change with a bone resorption marker (Table 1). This is called the coupling effect for bone metabolism, which is a local conjugate phenomenon of bone formation at the absorbed site in bone tissue. Such bone formation after bone resorption naturally causes a change in the bone metabolic marker $[19,20]$. Recently, severely suppressed bone turnover (SSBT) due to the fundamental effect of bisphosphonates has received attention. It has been reported that SSBT caused by the administration of bisphosphonates leads to a precursor state in fractures of the femur shaft (i.e., an atypical fracture); thus, careful observation of the bone metabolic marker is required [21]. Therefore, it is suggested that the anti-RANKL antibody would not cause excessive bone
Table 2. The change ratio of bone mass in terms of other osteoporosis drugs

\begin{tabular}{lcc} 
Characteristic & $\begin{array}{c}\text { The lumbar spine } \\
\text { YAM level (\%) }\end{array}$ & Periods (mo) \\
\hline Alendronate & 7.48 & $24-36$ \\
\hline Risedronate & 4.54 & $18-36$ \\
\hline Zoledronate & 6.71 & 36 \\
Raloxifene & 2.51 & 24 \\
\hline Teriparatide & 8.60 & 21 \\
\hline
\end{tabular}

YAM, young adult mean.

formation by the coupling effect, and the risk of an atypical fracture by SSBT could be lowered.

Furthermore, in this study, the bone density was significantly increased by the anti-RANKL antibody in our study. Reports on the change in the ratio of bone mass in terms of other osteoporosis drugs are shown in Table 2 $[6,22]$. Compared with the change in the ratio for other osteoporosis drugs, the rate indicated a good result-an average increased rate of $8.9 \%$, regardless of the shortterm follow-up or 6 months after treatment. Moreover, in a study on the effect of administering the anti-RANKL antibody over the long-term, it was reported that the change rate was $9.2 \%$, a higher improvement rate than for other osteoporosis drugs, therefore, a greater effect is expected with long-term administration [23].

We did not have any patients drop out of treatment during the 6-month study period. Difficulty in similar studies has been reported in general, such as not obtaining an actual sense of the treatment's effect or a high frequency rate for the occurrence of adverse effects in drug compliance [24].

However, as described previously, we estimated that patients could actually feel the treatment effect as the numerical values of their blood drawings and imaging studies improved. In addition, the average VAS level for 
back pain was temporally decreased before and after 1, 2, 3, and 6 months of treatment. Therefore, it can be surmised that the patients could feel a change in a specific symptom as their back pain improved. From this aspect, it is considered that the effect of anti-RANKL antibody treatment can be easily sensed, and no one in this study dropped out.

Furthermore, the anti-RANKL antibody does not have many adverse effects, such as heartburn from bisphosphonates that would lead to discontinuing treatment. The main adverse effect for the anti-RANKL antibody is hypocalcemia, but the frequency is relatively low $(0.8 \%)$ [25]. Moreover, hypocalcemia was maintained within the normal range before treatment and 1 week and 1 month after treatment for all cases in our study. Thus, it is likely that the high level of drug compliance resulted from the lack of adverse effects. However, we recommend the strict monitoring of hypocalcemia before treatment in all cases, and the administration of calcium and vitamin D may be effective in preventing hypocalcemia.

As for the study limitation, the follow-up period was short (i.e., 1 month for evaluating the bone metabolic markers and serum calcium levels and 6 months for evaluating drug compliance, the YAM level, and the back pain VAS). In the future, we plan to conduct a prospective study to examine the time course changes in bone metabolic markers, and to assess drug compliance over a longer follow-up period.

\section{Conclusions}

The average TRACP $5 b$ level was significantly lowered by the administration of the anti-RANKL antibody, but there was no significant difference in the average P1NP level. The results from our study suggest that the administration anti-RANKL antibody suppresses bone resorption, and maintains bone formation.

\section{Conflict of Interest}

We disclose COI with the following company: Daiichi Sankyo Company, limited.

\section{References}

1. Chin DK, Park JY, Yoon YS, et al. Prevalence of osteoporosis in patients requiring spine surgery: incidence and significance of osteoporosis in spine disease. Osteoporos Int 2007;18:1219-24.

2. Orriss IR, Key ML, Colston KW, Arnett TR. Inhibition of osteoblast function in vitro by aminobisphosphonates. J Cell Biochem 2009;106:109-18.

3. Bolland MJ, Grey A, Avenell A, Gamble GD, Reid IR. Calcium supplements with or without vitamin D and risk of cardiovascular events: reanalysis of the Women's Health Initiative limited access dataset and meta-analysis. BMJ 2011;342:d2040.

4. Martino S, Disch D, Dowsett SA, Keech CA, Mershon JL. Safety assessment of raloxifene over eight years in a clinical trial setting. Curr Med Res Opin 2005;21:1441-52.

5. Layton D, Clarke A, Wilton LV, Shakir SA. Safety profile of raloxifene as used in general practice in England: results of a prescription-event monitoring study. Osteoporos Int 2005;16:490-500.

6. Black DM, Kelly MP, Genant HK, et al. Bisphosphonates and fractures of the subtrochanteric or diaphyseal femur. N Engl J Med 2010;362:1761-71.

7. Khosla S, Burr D, Cauley J, et al. Bisphosphonateassociated osteonecrosis of the jaw: report of a task force of the American Society for Bone and Mineral Research. J Bone Miner Res 2007;22:1479-91.

8. Yoneda T, Hagino H, Sugimoto T, et al. Bisphosphonate-related osteonecrosis of the jaw: position paper from the Allied Task Force Committee of Japanese Society for Bone and Mineral Research, Japan Osteoporosis Society, Japanese Society of Periodontology, Japanese Society for Oral and Maxillofacial Radiology, and Japanese Society of Oral and Maxillofacial Surgeons. J Bone Miner Metab 2010;28:365-83.

9. Bashutski JD, Eber RM, Kinney JS, et al. Teriparatide and osseous regeneration in the oral cavity. $\mathrm{N}$ Engl J Med 2010;363:2396-405.

10. Cheung A, Seeman E. Teriparatide therapy for alendronate-associated osteonecrosis of the jaw. N Engl J Med 2010;363:2473-4.

11. Lamke B, Sjoberg HE, Sylven M. Bone mineral content in women with Colles' fracture: effect of calcium supplementation. Acta Orthop Scand 1978;49:143-6.

12. Solomon DH, Avorn J, Katz JN, et al. Compliance with osteoporosis medications. Arch Intern Med 2005;165:2414-9.

13. Matsumoto T, Ito M, Hayashi $Y$, et al. A new active vitamin D3 analog, eldecalcitol, prevents the risk of 
osteoporotic fractures: a randomized, active comparator, double-blind study. Bone 2011;49:605-12.

14. Morii H, Ohashi Y, Taketani Y, et al. Effect of raloxifene on bone mineral density and biochemical markers of bone turnover in Japanese postmenopausal women with osteoporosis: results from a randomized placebo-controlled trial. Osteoporos Int 2003;14:793800.

15. Itabashi A, Yoh K, Chines AA, et al. Effects of bazedoxifene on bone mineral density, bone turnover, and safety in postmenopausal Japanese women with osteoporosis. J Bone Miner Res 2011;26:519-29.

16. Uchida S, Taniguchi T, Shimizu T, et al. Therapeutic effects of alendronate $35 \mathrm{mg}$ once weekly and $5 \mathrm{mg}$ once daily in Japanese patients with osteoporosis: a double-blind, randomized study. J Bone Miner Metab 2005;23:382-8.

17. Kishimoto H, Fukunaga M, Kushida K, et al. Efficacy and tolerability of once-weekly administration of 17.5 $\mathrm{mg}$ risedronate in Japanese patients with involutional osteoporosis: a comparison with 2.5-mg once-daily dosage regimen. J Bone Miner Metab 2006;24:40513.

18. Tokuyama N, Tanaka S. Updates of denosumab, antiRANKL antibody for osteoporosis. Clin Calcium 2014;24:85-91.

19. Nakamura M, Udagawa N, Matsuura S, et al. Osteoprotegerin regulates bone formation through a cou- pling mechanism with bone resorption. Endocrinology 2003;144:5441-9.

20. Parfitt AM. The mechanism of coupling: a role for the vasculature. Bone 2000;26:319-23.

21. Odvina CV, Zerwekh JE, Rao DS, Maalouf N, Gottschalk FA, Pak CY. Severely suppressed bone turnover: a potential complication of alendronate therapy. J Clin Endocrinol Metab 2005;90:1294-301.

22. Cranney A, Guyatt G, Griffith L, Wells G, Tugwell P, Rosen C. Meta-analyses of therapies for postmenopausal osteoporosis. IX: Summary of meta-analyses of therapies for postmenopausal osteoporosis. Endocr Rev 2002;23:570-8.

23. Cummings SR, San Martin J, McClung MR, et al. Denosumab for prevention of fractures in postmenopausal women with osteoporosis. N Engl J Med 2009;361:756-65.

24. Cortet B, Benichou O. Adherence, persistence, concordance: do we provide optimal management to our patients with osteoporosis? Joint Bone Spine 2006;73:e1-7.

25. Nakamura T, Matsumoto T, Sugimoto T, et al. Clinical Trials Express: fracture risk reduction with denosumab in Japanese postmenopausal women and men with osteoporosis: denosumab fracture intervention randomized placebo controlled trial (DIRECT). J Clin Endocrinol Metab 2014;99:2599-607. 